\title{
Using Modelica to Assess the Resilience of a Heat Supply System
}

\author{
Anne Senkel, Gerhard Schmitz \\ Institute of Engineering Thermodynamics, Hamburg University of Technology, \\ Denickestr. 17, 21073 Hamburg, Germany, \\ \{anne.senkel, schmitz\}@tuhh.de
}

\begin{abstract}
With the rising importance of resilience in today's energy discussions, this paper uses a quantitative method to evaluate the resilience of energy supply systems. For this purpose, a suitable assessment method is introduced and applied to a heat supply system of a one family dwelling to which disturbances are induced. It can be shown that both room temperature and delivered heat flow can be used to calculate and evaluate a resilience index using the system answer's maximum deviation, recovery time and performance loss. While the first reflects the actual service offering, the second approach assesses the system's reaction and balancing effort.
\end{abstract}

\section{Introduction}

Current discussions about the existing and the future energy supply system mainly focus on two topics: cost and $\mathrm{CO}_{2}$ emission. However, a third issue is gaining importance: the resilience of an energy supply system.

Investigating resilience leads to considering a wide field of research since various areas of science use this term to describe material, human or system behavior. As different as these fields might be, all of them use resilience (lat. resilire: jump back, rebound, return) to describe the ability to resist and react to known and unknown disturbances and to return into a stable state afterwards.

One of the most influential researchers in this field is Crawford S. Holling who investigated the topic from an ecological view. In 1973, he introduced the expression engineering resilience which defines resistance and speed of return to the equilibrium as measures of resilience. Further, he established the term ecosystem resilience which focuses on systems that are not characterized by one equilibrium steady-state. This type of resilience is hence described "by the magnitude of disturbance that can be absorbed before the system changes its structure by changing the variables and processes that control behavior." (Gunderson and Holling, 2012). While the efficiency of the system's functionality plays a key role for the engineering resilience, the ecological resilience focuses on the existence of a system's functionality.

In the project nordwest 2050 (Fichter et al., 2010), guiding principles for resilient energy supply systems are developed based on the concepts of Holling and Gunderson. They therefore introduce design features of resilient systems and organize them as system capacities and system structures. Capacities that help increasing a system's resilience are:

- adaptive capacity

- resistive capacity

- formative capacity

Whereas system structures that enhance resilience are:

- diversity

- redundancy

- buffer capacities

- back coupling mechanisms

- dampers

While Fichter et al. (2010) present qualitative evaluations methods, another perspective that focuses on a quantitative framework, has been developed in the field of disaster research (Cimellaro et al., 2010). For this purpose, a non-dimensional functionality function is used, and resilience defined as the normalized area underneath the curve of this function (cf. Figure 1). Moreover, Cimellaro et al. (2010) conduct their method to assess the resilience of a hospital network concerning earthquake disasters.

This method of evaluating a system's functionality and its time-dependent behavior is progressed by Francis and Bekera (2014) and Nan and Sansavini (2017). Rather than evaluating the area underneath the functionality, they assess the system's rapidity, recovery time and performance loss. While Francis and Bekera (2014) also implement a vulnerability analysis and the system's fragility in their considerations, Nan and Sansavini (2017) conduct a multi-layer approach by examining human operators, control systems and the system itself.

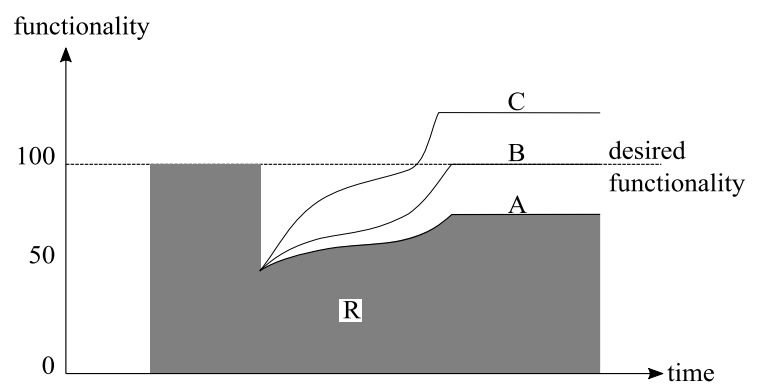

Figure 1. Resilience $(R)$ definition according to Cimellaro et al. (2010), A: functionality loss, B: functionality recovery, $C$ : functionality enhancement. 


\section{Methods}

As it was shown in the previous section, the definition of resilience is not yet fully concluded since different research fields approach this topic differently leading to different emphases. The following chapter presents a method developed by Senkel et al. (2019) which allows the resilience assessment of energy supply systems modelled in Modelica ${ }^{\circledR}$ (Modelica Association, 2019). Therefore, the quantitative approaches of Francis and Bekera (2014) and Nan and Sansavini (2017) were adapted.

Since system functionality is not a physical value that can be measured, a characteristic value that reflects a system's functionality, is determined. This value depends on the considered system. For an electric grid, the grid frequency could be such a value. In case of a heating system the room temperature could be used, and the gas sector could be evaluated by using the operating pressure. The following considerations only imply the use of one of these values for the assessed system. However, it is also conceivable to evaluate more than one value to gain a more detailed resilience analysis. For each value, a tolerance band can be defined. Deviations within this tolerance band are considered acceptable and without any major impact on the continuous supply of the system's services. For example, a heating system's service (supplying enough heat so people do not feel cold) is maintained even though the room temperature slightly drops underneath the set point. Note that this set point is not necessarily constant.

A system's characteristic value may react to a deviation as shown in Figure 2. Deviations that remain within the tolerance band, indicate a resilient system (Figure 2, left). When a disturbance leads to a collapse of the system, its characteristic value will not return into the tolerance band (Figure 2, right). Therefore, this behavior is considered as irresilient. Between these borderline cases, several system answers with different characteristics can occur (Figure 2, middle). To compare these characteristics, the maximum deviation, the recovery time, and the performance loss are evaluated.

The maximum deviation $M D$ which reflects a system's resistive capacity, is defined with the maximum difference $\Delta x$ between the borders of the tolerance band $\left(x_{\min }, x_{\max }\right)$ and the characteristic value $x$ when it leaves the tolerances:

$$
\Delta x= \begin{cases}x-x_{\max } & \text { if } x \geq x_{\max } \\ 0 & \text { if } x_{\min }<x<x_{\max } \\ x_{\min }-x & \text { if } x \leq x_{\min }\end{cases}
$$

Since the resilience index aims on comparing different systems with each other, every considered element is normalized to gain a non-dimensional figure. Therefore $x_{n o r m}$ is introduced to define the maximum deviation:

$$
M D=\frac{\Delta x_{\max }}{\Delta x_{\text {norm }}}
$$

The recovery time $R T$, a measure for the restorative capacity, is defined as the time span between the point of time $t_{\mathrm{d}}$ when the indicator value first leaves the tolerance band and the point $t_{\mathrm{r}}$ when it remains permanently within the tolerances, normalized by $\Delta t_{\text {norm }}$ :

$$
R T=\frac{t_{\mathrm{r}}-t_{\mathrm{d}}}{\Delta t_{\mathrm{norm}}}
$$

The performance loss $P L$ describes the normalized area between tolerance band and the course of the characteristic figure (depicted as $A$ in Figure 2):

$$
P L=\frac{\int_{t_{\mathrm{d}}}^{t_{\mathrm{r}}} \Delta x d t}{A_{\text {norm }}}
$$

Note that the presented normalization values can be interpreted as the maximum values of deviation that are still considered as resilient. Hence, it is recommended to choose sensible values to gain reasonable results and avoid unnecessary arbitrariness. Multiplying these three resilience elements leads to the irresilience index IRI:

$$
I R I=R \cdot M D \cdot P L
$$

From which the resilience index $R I$ can be derived:

$$
R I=\frac{1}{1+I R I}
$$

With this, we define $R I=1$ for resilient and $R I=0$ for irresilient system reactions (cf. Figure 2). This general approach can be transferred to any other energy system if a suitable physical value is available. Furthermore, a tolerance band and reasonable normalization values need to be found.
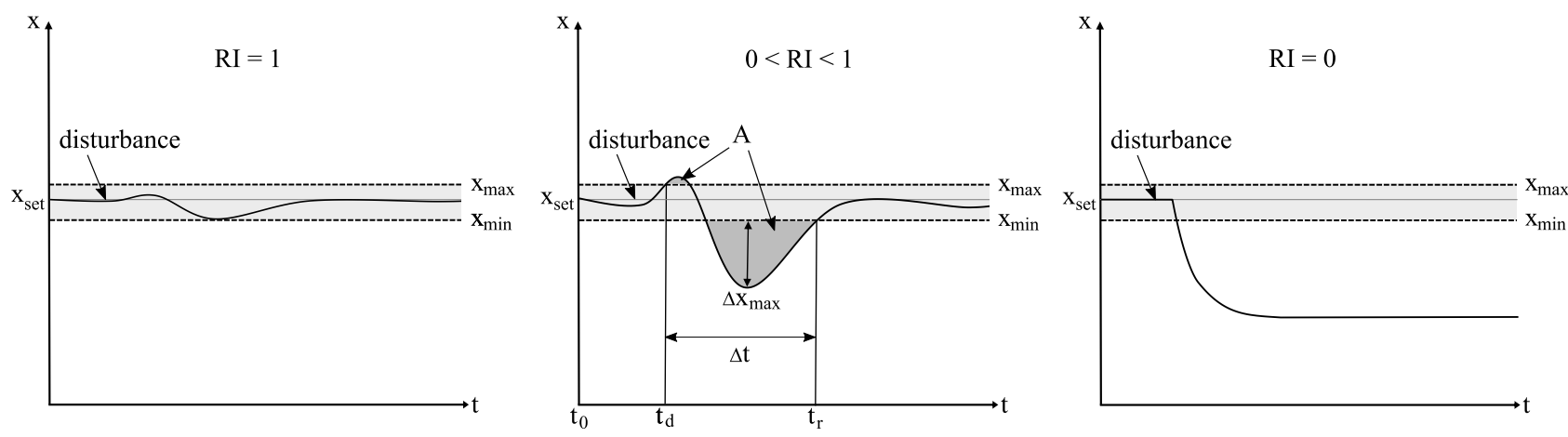

Figure 2: Possible system reactions to disturbances: remaining within tolerances (left), leaving and returning into tolerances (middle), permanently leaving tolerances (right) 


\section{Use Case}

The resilience index presented in the previous chapter was used by Senkel et al. (2019) to evaluate a heating system of a single family house (SFH) using the room temperature as resilience indicator. Therefore, Modelica (Modelica Association, 2019), an acausal, open-source, object-oriented, equation-based programming language, was used. Since Modelica allows a physically based, dynamic simulation of the heating system, the dynamic reactions to disturbances can be modelled and assessed. In the following chapter, another heating system is evaluated, further disturbances are introduced and the possibility to use the supply temperature or rather the delivered heat flow as resilience indicator is examined.

\section{Model Structure}

Therefore, a validated dynamic low order model of the heat losses of an SFH (Senkel, 2017) is integrated in a heating system modelled with the TransiEnt Library (Andresen et al., 2015) and the ClaRa Library (Brunnemann et al., 2012) using the Modelica environment Dymola (Dassault Systèmes, 2018). The structure of the model is shown in Figure 3.

The considered heating system is reduced to only provide hot water for heating purposes. The generation of domestic hot water is not investigated. The heating water is produced by a heat pump and stored in a buffer storage. The mass flow through the heat pump is regulated by its supply pump to attain a heat pump outlet temperature of $55^{\circ} \mathrm{C}$. The heat pumps power is controlled to reach $50{ }^{\circ} \mathrm{C}$ in the upper part of the buffer storage. At the consumer side, the supply temperature is regulated according to a heating curve by a mixing valve. The room temperature is adjusted by a thermostat whose set point is $22{ }^{\circ} \mathrm{C}$ (according to EN 15251 (European Commitee for Standardization, 2007)). In Table 1, the parameters of the heating system are collected. Note that this model is built to generate plausible results to be able to test the introduced evaluation method since this is the focus of the paper. Therefore, validated component models are utilized, and the results are checked for plausibility. However, no system validation is conducted.

The following results are simulated with the solver Dassl in a 15-minutes resolution with a tolerance of 0.0001 .

Table 1: Parameters of the house and the heating system

\begin{tabular}{lr}
\hline Parameter & Value \\
\hline Nominal supply temperature & $45{ }^{\circ} \mathrm{C}$ \\
Room temperature set point & $22{ }^{\circ} \mathrm{C}$ \\
Nominal mass flow & $0.2 \mathrm{~kg} / \mathrm{s}$ \\
Nominal pressure & $1 \mathrm{bar}$ \\
Nominal heat flow of heat pump & \\
(A2/W35) & $8.33 \mathrm{~kW}^{1}$ \\
Building standard & EnEV $2014^{2}$ \\
Floor area & $100 \mathrm{~m}^{2}$ \\
Window area & $16 \mathrm{~m}^{2}$ \\
Minimal outdoor temperature & $-12{ }^{\circ} \mathrm{C}$ \\
\hline
\end{tabular}

\section{Scenarios}

Figure 3 shows several disturbances which were introduced into the system. All presented disturbances are simulated with the weather data of Hamburg between January 30 $0^{\text {th }}$ and February $2^{\text {nd }} 2012$ (Lange, 2014). Furthermore, all disturbances are set to occur in the same time interval (first twelve hours at the beginning of February $1^{\text {st }}$ ). On the consumer side, the pump's mass flow is set to zero which models a pump or pump control failure (D1). Disturbance D2 models a closed thermostat in the considered period which can occur due to mechanical faults of the valve. At the producer side, the mass flow of the supply pump is also set to zero (D3) and finally the heat pump's heat production is set to zero (D4). Both disturbances can occur due to faults in the components or their control.

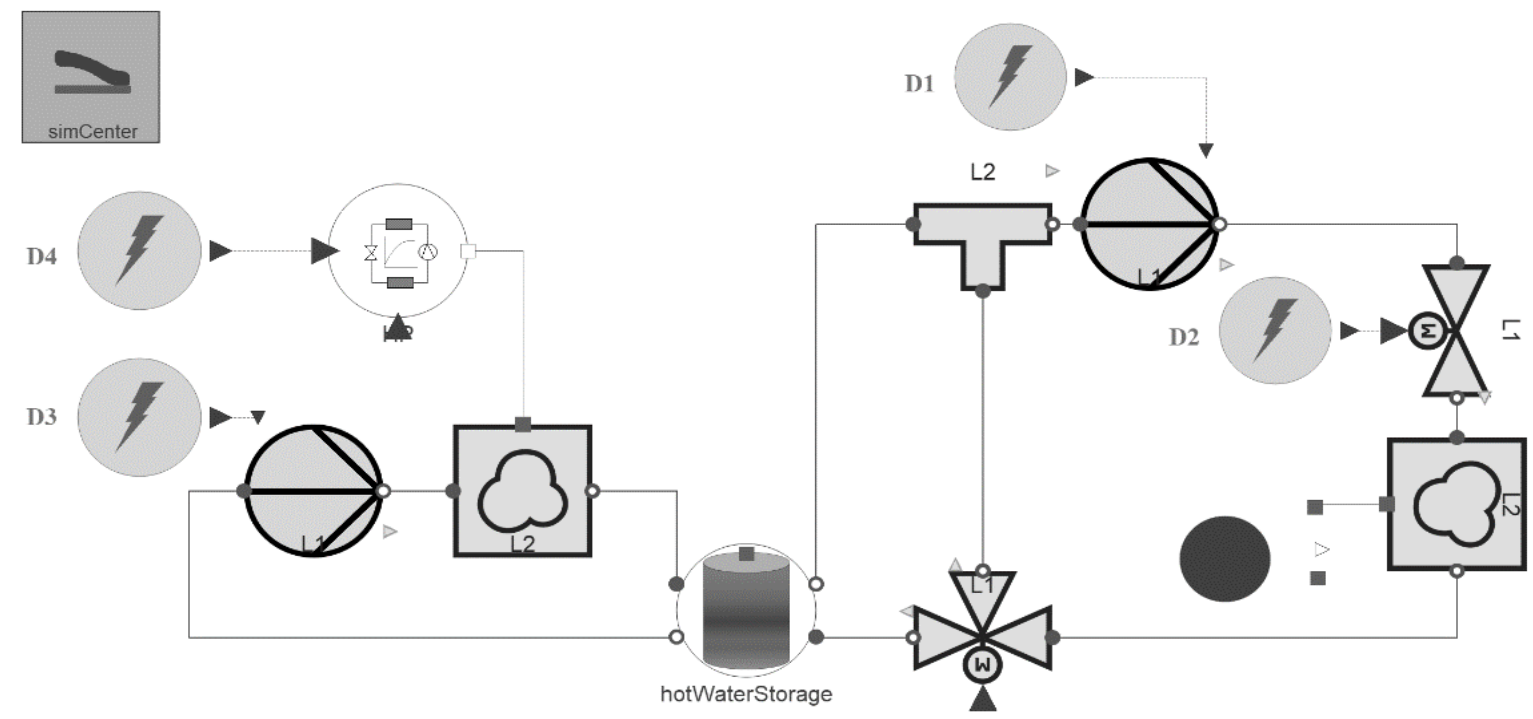

Figure 3: Investigated heat system with implemented disturbances
${ }^{1}$ STIEBEL ELTRON GmbH \& Co. KG (2019).
${ }^{2}$ Bundesregierung Deutschland (2013). 


\section{Results}

\section{Disturbances on the Consumer Side}

When investigating the system's reaction to the failure of the pump and the thermostat, one notices that the considered room and supply temperature and the delivered heat flow behave in the same manner (cf. Figure 4). This is because both disturbances have the same effect on the system, they stop the mass flow through the radiator and therefore the heat supply to the building. For the purpose of simplicity, only the results of D1 will be evaluated in the following discussion.

When looking at the profiles of the room and the supply temperature, one notices that even though the supply temperature only slightly drops, the room temperature significantly deviates from the set point. The reason for this behavior is that the room temperature is dependent on the heat delivered to the room. This heat flow drops to almost zero (c.f. Figure 4) because the mass flow is disturbed in the considered scenarios. The supply temperature itself drops only slightly due to heat losses to the surroundings. Apart from that, the supply temperature would be sufficiently high to heat the room since the producer side is not disturbed.
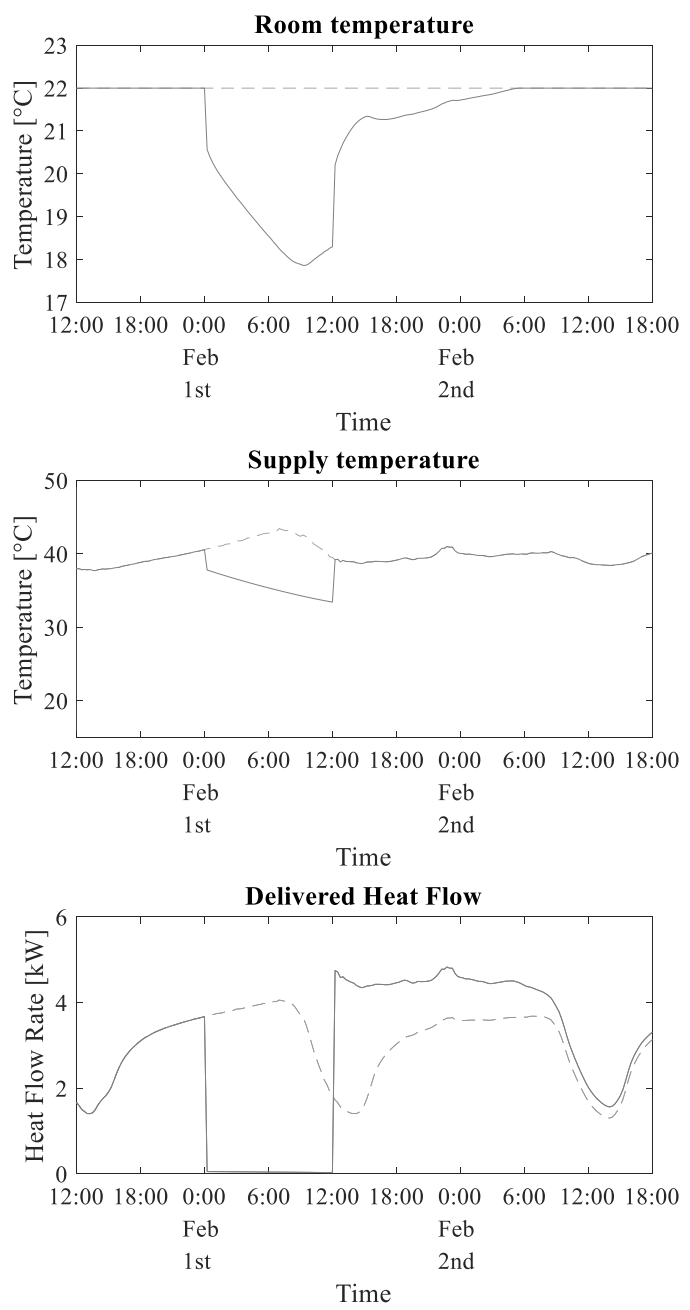

Figure 4: Room temperature, supply temperature and heat flow during disturbances D1 and D2 (dashed: undisturbed, solid: D1 and D2)

\section{Disturbances on the Producer Side}

For the disturbances on the producer side, a similar picture emerges. Since the pump's control is connected with the heat output of the heat pump and the heat pump's control prevents it from overheating, in both disturbance cases the mass flow rate in the producer circuits becomes zero. Therefore, the temperature and heat flow profiles in both disturbance scenarios are identical and the following discussion will focus on the results of disturbance D3.

In these scenarios, the supply temperature sinks significantly due to the disturbances on the production side. Because of the buffer storage and the undisturbed mass flow through the radiator, the delivered heat flow does not drop as fast as in the disturbance scenarios D1 and D2.

Especially in comparison with the results of the first two disturbances, it becomes clear that the supply temperature as sole resilience indicator will not be sufficient. Therefore, the heat flow rate will be used to calculate the resilience indices and the obtained results will be compared with the indices calculated using the room temperature.
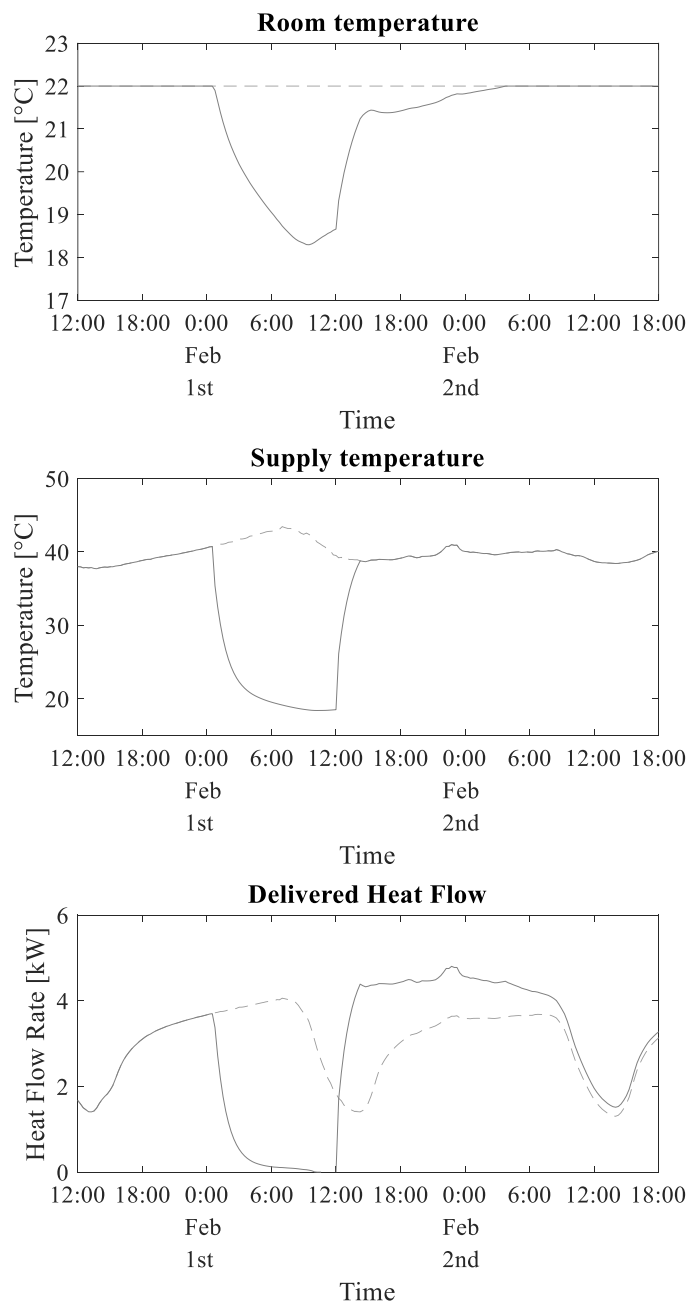

Figure 5: Room temperature, supply temperature and heat flow during disturbances D3 and D4 (dashed: undisturbed, solid: D3 and D4) 


\section{Parameter Study for Disturbance D3}

To be able to assess a broader range of resilience indices, for disturbance D3 the volume of the buffer storage is varied $\left(V_{\text {sto }}=\{0.2,0.4,0.6,0.8,1.0,1.2,1.4,1.6\} \mathrm{m}^{3}\right)$. Disturbance D1 is not part of the parameter study since the size of the buffer storage has only a small influence on the system's answer when the consumer mass flow is disabled. The profiles of the room temperature and the heat flow are depicted in Figure 6. For reasons of clarity, only every second result is shown. Additionally, the tolerance band (c.f. Figure 2) is marked $\left(\Delta T_{\text {tol }}= \pm 1 \mathrm{~K}\right.$, $\left.\Delta \dot{Q}_{\text {tol }}= \pm 1 \mathrm{~kW}\right)$. The set point of the room temperature is $22{ }^{\circ} \mathrm{C}$ (c.f. Model Structure). For the delivered heat flow, the undisturbed system is simulated and the occurring heat flow is used as set point. As expected, the temperature and heat flow deviations become less with increasing storage size.
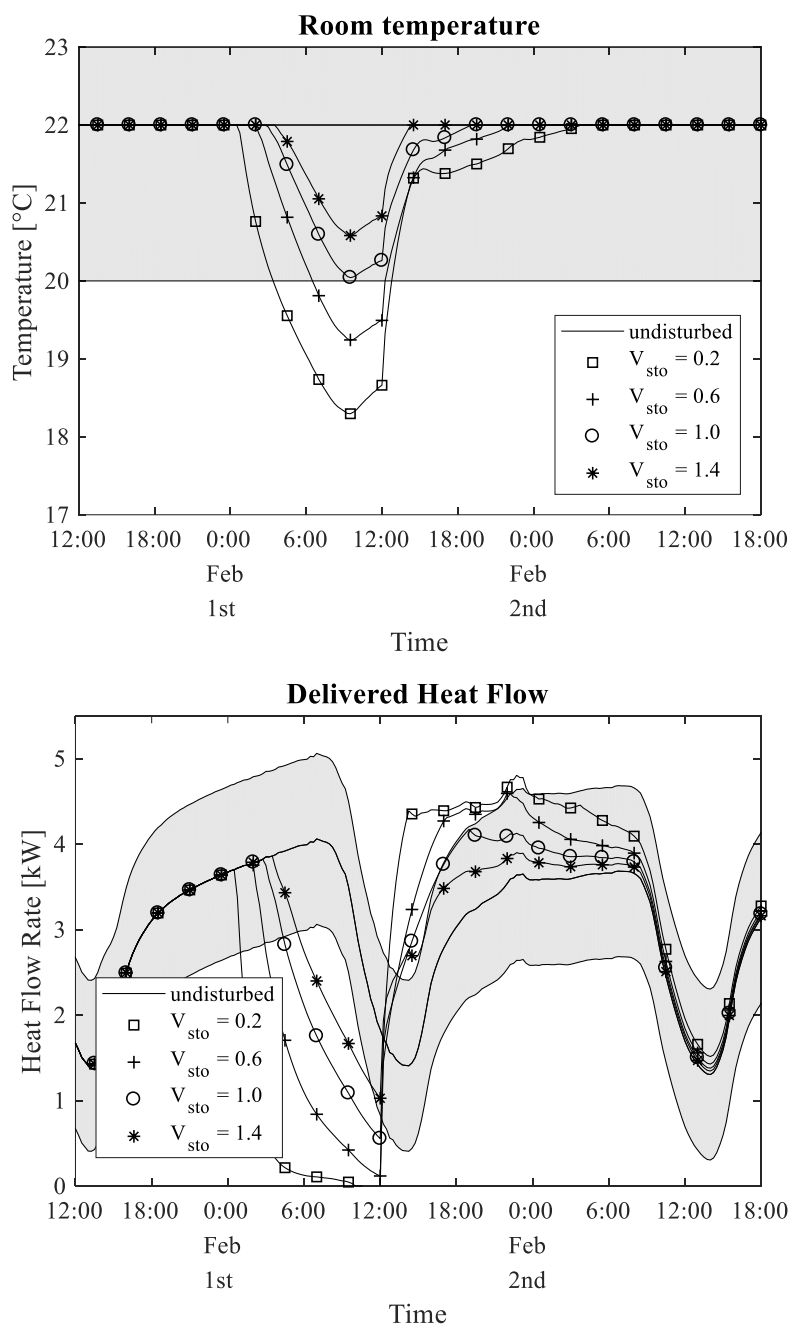

Figure 6: Room temperature and heat flow profiles of D3 with different buffer storages (grey: tolerance band)

\section{Resilience Indices}

According to the evaluation method introduced in the second section, the resilience indices for the disturbances D1 and D3 are computed and listed in Table 2. The results for the parameter studies are presented in Table 3. For the purpose of clarity, only every second result is shown. The normalization values for the resilience indices calculated with the room temperature are $\Delta x_{\text {norm }}=1 \mathrm{~K}$ and $\Delta t_{\text {norm }}=6 \mathrm{~h}$. For the resilience indices that utilize the delivered heat as indicator, $\Delta x_{\text {norm }}=1 \mathrm{~kW}$ and $\Delta t_{\text {norm }}=24 \mathrm{~h}$ was used. In both calculations $A_{\text {norm }}=$ $\Delta x_{\text {norm }} \cdot \Delta t_{\text {norm }}$ is applied.

Table 2: Resilience indices of disturbances D1 and D3

\begin{tabular}{|c|c|c|c|c|c|}
\hline \multirow{2}{*}{\multicolumn{2}{|c|}{ Indicator }} & \multicolumn{2}{|c|}{ D1 } & \multicolumn{2}{|c|}{ D3 } \\
\hline & & $T_{\mathrm{R}}$ & $\dot{Q}_{d e l}$ & $T_{\mathrm{R}}$ & $\dot{Q}_{\mathrm{del}}$ \\
\hline \multicolumn{2}{|c|}{$\Delta x_{\max }[\mathrm{K}] /[\mathrm{kW}]$} & 2.1 & 3.0 & 1.7 & 3.0 \\
\hline \multicolumn{2}{|c|}{$\Delta t[\mathrm{~h}]$} & 10.7 & 24.0 & 9.5 & 23.0 \\
\hline \multicolumn{2}{|c|}{$P L[\mathrm{Kh}] /[\mathrm{kWh}]$} & 14.9 & 39.8 & 10.5 & 31.9 \\
\hline \multirow[t]{2}{*}{$R I$} & wo/ PL & 0.21 & 0.25 & 0.27 & 0.26 \\
\hline & $\mathrm{w} / \mathrm{PL}$ & 0.17 & 0.17 & 0.30 & 0.21 \\
\hline
\end{tabular}

\section{Discussion}

When looking at the resilience indices, it becomes obvious that they depend, as expected, on the severity and time duration of the indicator value's deviation. Also, it is noticeable that the location of the disturbance has an impact on the index. While the failure of the heat production pump (D3) is largely absorbed by the heat capacity of the buffer storage, the failure of the consumer pump (D1) right in front of the heat transfer leads to a stronger loss of functionality. Additionally, one can observe that the resilience indices computed with the room temperature differ stronger from each other than the ones calculated with the delivered heat flow. This effect can be led back to the different considered indicators, there absolute values and the differences between them. For example, the slower decline of delivered heat in D3 does lead to higher room temperature than in D1 even though the heat flow profiles do not differ very much, especially when regarding the recovery phase after the disturbance.

The consideration of the performance loss leads to an additional weighting of the severity of the deviation. Since small deviations barely lead to a surpassing of $A_{\text {norm }}$, the factor $\int_{t_{\mathrm{d}}}^{t_{\mathrm{r}}} \Delta x d t / A_{\text {norm }}$ becomes less than 1 and therefore leads to a higher resilience index than without the consideration of $P L$ (c.f. Table 3). The opposite occurs when very large deviations arise. Since $\int_{t_{\mathrm{d}}}^{t_{\mathrm{r}}} \Delta x d t$ exceeds $A_{n o r m}$ in these cases, the resilience index is additionally lowered leading to an extra punishment of large deviations (c.f. Table 2). This behavior is welcomed since the effects of small deviations from the set values are barely noticeable for the consumers while high deviations can additionally lead to effects on health. 
Table 3: Resilience indices of the system under disturbance D3 with an enhanced buffer storage

\begin{tabular}{|c|c|c|c|c|c|c|c|c|}
\hline & \multicolumn{2}{|c|}{$V_{\text {sto }}=0.2 \mathrm{~m}^{3}$} & \multicolumn{2}{|c|}{$V_{\text {sto }}=0.6 \mathrm{~m}^{3}$} & \multicolumn{2}{|c|}{$V_{\text {sto }}=1.0 \mathrm{~m}^{3}$} & \multicolumn{2}{|c|}{$V_{\text {sto }}=1.4 \mathrm{~m}^{3}$} \\
\hline Indicator & $\overline{T_{\mathrm{R}}}$ & $\dot{Q}_{\text {del }}$ & $\overline{T_{\mathrm{R}}}$ & $\dot{Q}_{\text {del }}$ & $T_{\mathrm{R}}$ & $\dot{Q}_{\mathrm{del}}$ & $T_{\mathrm{R}}$ & $\dot{Q}_{\mathrm{del}}$ \\
\hline$\Delta x_{\max }[\mathrm{K}] /[\mathrm{kW}]$ & 1.7 & 3.0 & 0.8 & 2.4 & - & 1.6 & - & 1.0 \\
\hline$\Delta t[\mathrm{~h}]$ & 9.5 & 23.0 & 5.8 & 19.3 & - & 12.3 & - & 10.0 \\
\hline$P L[\mathrm{Kh}] /[\mathrm{kWh}]$ & 10.5 & 31.9 & 3.0 & 17.7 & - & 8.3 & - & 3.7 \\
\hline wo/ PL & 0.27 & 0.26 & 0.58 & 0.35 & 1 & 0.56 & 1 & 0.72 \\
\hline $\mathrm{w} / \mathrm{PL}$ & 0.30 & 0.21 & 0.85 & 0.42 & 1 & 0.78 & 1 & 0.94 \\
\hline
\end{tabular}

When assessing the results of the parameter study and comparing the resilience indices that are derived from the room temperature, with the ones derived from the heat flow to the consumer, one notices that the first rise faster with increasing storage size ( Figure 7). On the one hand, this behavior can be explained by the different normalization values. By adapting $x_{\text {norm }}$ and $\Delta t_{\text {norm }}$, one could achieve a better correlation of the indices. However, this adaptation can only be conducted to a certain extent since too large normalization values will lead to very high resilience indices whose informative values are very small.

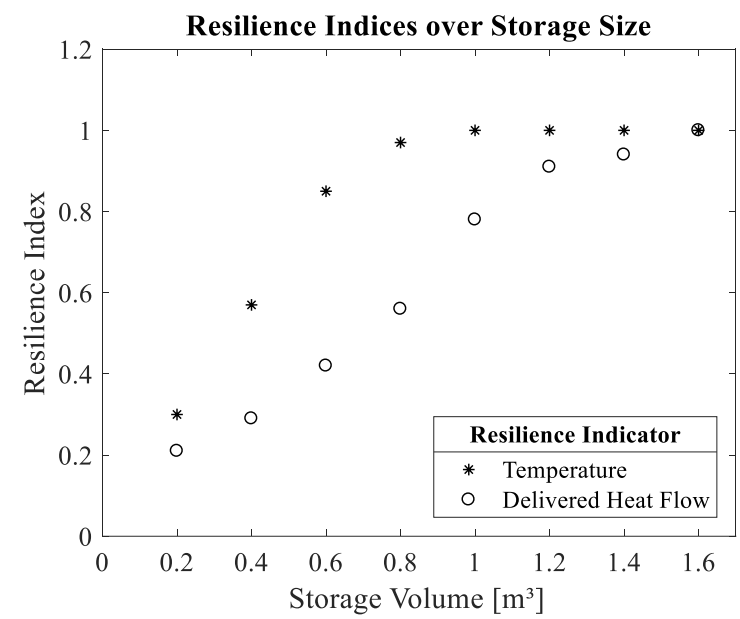

Figure 7: Resilience indices in dependence to storage size

Another explanation can be given by understanding that the heat flow to the consumer reflects the system's reaction more than the room temperature. While the room temperature mirrors the actual service offering of the heating system, providing enough heat to achieve a comfortable room temperature, the heat flow shows the heating system's effort of supplying the demanded heat and reheating the building after the temperature drop. Therefore, the restorative phase of the system is represented in detail which can be retraced by regarding the higher heat flow after the disturbance period when the house is heated up.

One may conclude that installing a buffer storage of $1.6 \mathrm{~m}^{3}$ leads to a resilient system. However, it must be kept in mind that the other disturbance scenario (D1) still causes a system answer outside the tolerance band. Therefore, it is important to assess several disturbances to find system improvements that have a positive impact in as many as possible cases. Additionally, every system improvement needs to be rated by further factors as financial efficiency, practicability, and importance of the systems. For example, one may deservedly consider a $1.6 \mathrm{~m}^{3}$ buffer storage a quite expensive and space consuming investment in the resilience of a heating system that supplies heat for only one family.

\section{Conclusion}

In this paper, a method of evaluating the resilience of a heat supply system with Modelica is presented and applied to a simple example. The structure of a resilience index is introduced and the index itself computed for the presented use case of a heating system of a single family house. For the resilience analysis, two indicators, the room temperature and the delivered heat flow rate, were used.

It can be shown that the room temperature and the delivered heat flow can be used to determine the resilience index and therefore evaluate a heating system's resilience. However, it also became obvious that the different indicators produce different scales. Therefore, the boundary conditions have to be similar when comparing results.

While using the room temperature results in an assessment of the human comfort and therefore the actual service offering, the utilization of the delivered heat flow leads to the evaluation of the system's reaction and the measures taken to return into a stable state. Therefore, this approach is recommended when the consequences for the system are to be stressed as well.

Since the applied approach focuses on characteristic values of the considered energy supply system, it is possible to transfer it to any other system, regardless of the surrounding conditions or the system structure. It is important, however, to be able to detect an indicator whose deviation from its set point is able to reflect the system's answer to disturbances. For example, Heckel and Becker (2019) apply the same approach to assess the resilience using the frequency and a bus voltage in an electric grid.

By quantifying resilience, it is possible to determine a "cost" of resilience. Hence, the increase of resilience by a certain percentage can be compared with the increase of $\mathrm{CO}_{2}$ emissions or the arising additional costs. Therefore, 
existing frameworks can be extended by incorporating the presented method.

It is assumed that evaluating the heat flow deviations is an interesting approach for assessing heat networks as well, especially, when including the provision of hot drinking water. Hence, future work should intend on applying both presented approaches on thermal networks. In this regard, one also needs to find an effective way on dealing with the fact that thermal networks supply several households with heat and therefore a method needs to be found that allows the assessment of the different consequences one disturbance can have on different consumers. In this context, the introduction of global and local resilience indices is assumed to be sensible.

\section{Acknowledgements}

The authors greatly acknowledge the funding from the German Federal Ministry of Economic Affairs and Energy for the project "ResiliEntEE - Resilienz gekoppelter Energienetze mit hohem Anteil Erneuerbarer Energien" (ResilientEE - Resilience of integrated energy networks with a high share of renewable energies, project number: 03ET4048).

\section{References}

Andresen, L., Dubucq, P., Peniche Garcia, R., Ackermann, G., Kather, A. and Schmitz, G. (2015), "Status of the TransiEnt Library: Transient Simulation of Coupled Energy Networks with High Share of Renewable Energy", in Modelica Association (Ed.), Proceedings Modelica Conference 2015, September 21-23, 2015, Linköping University Electronic Press, pp. 695-705.

Brunnemann, J., Gottelt, F., Wellner, K., Renz, A., Thüring, A., Roeder, V., Hasenbein, C., Schulze, C., Schmitz, G. and Eiden, J. (2012), "Status of ClaRaCCS: Modelling and Simulation of Coal-Fired Power Plants with CO2 Capture", in Proceedings of the 9th International MODELICA Conference, September 3-5, 2012, Munich, Germany, Sept. 3-5, 2012, Linköping University Electronic Press, pp. 609-618.

Bundesregierung Deutschland (2013), Zweite Verordnung zur Änderung der Energieeinsparverordnung: EnEV 2014.

Cimellaro, G.P., Reinhorn, A.M. and Bruneau, M. (2010), "Framework for analytical quantification of disaster resilience", Vol. 32 No. 11, pp. 3639-3649.

Dassault Systèmes (2018), "Dymola®”, available at: https://www.3ds.com/de/produkte-undservices/catia/produkte/dymola/.

European Commitee for Standardization (2007), EN 15251 2012-12, Indoor environmental input parameters for design and assessment of energy performance of buildings addressing indoor air quality, thermal environment, lighting and acoustics, Vol. 91.140.01, Brüssel.
Fichter, K., Gleich, A.v., Pfriem, R. and Siebenhüner, B. (2010), Theoretische Grundlagen für erfolgreiche Klimaanpassungsstrategien, Bremen.

Francis, R. and Bekera, B. (2014), “A metric and frameworks for resilience analysis of engineered and infrastructure systems", Reliability Engineering \& System Safety, Vol. 121, pp. 90-103.

Gunderson, L.H. and Holling, C.S. (2012), Panarchy: Understanding transformations in human and natural systems, Island Press, [United States].

Heckel, J.-P. and Becker, C. (2019), "Dynamic Simulation of an Integrated Energy System for Northern Germany with Improved Resilience", paper presented at International ETG Congress 2019, Esslingen am Neckar,Germany.

Lange, I. (2014), “Outdoor Temperatures, Hamburg Billwerder, 900s, 2012", available at: https://wettermast.uni-hamburg.de/.

Modelica Association (2019), "Modelica", available at: https://www.modelica.org.

Nan, C. and Sansavini, G. (2017), “A quantitative method for assessing resilience of interdependent infrastructures", Reliability Engineering \& System Safety, Vol. 157, pp. 35-53.

Senkel, A. (2017), "Vergleich verschiedener Arten der Wärmeverbrauchsmodellierung in Modelica", Master Thesis, Institute for Engineering Thermodynamics, Hamburg University of Technology, Hamburg, 09/2017.

Senkel, A., Bode, C. and Schmitz, G. (2019), "Evaluating the Resilience of Energy Supply Systems at the Example of a Single Family Dwelling Heating System", paper presented at 13th International Modelica Conference, March, 4-6, Regensburg, Germany.

STIEBEL ELTRON GmbH \& Co. KG (2019), "Tecnisches Datenblatt WPL 25 A", available at: https://www.stiebel-eltron.de/de/home/produkteloesungen/erneuerbare_energien/waermepumpe/luftwasserwaermepumpen/wpl_15_20_25_ac_s/wpl_25_a.htm 1. 